\title{
Cluster based wireless sensor network for forests environmental monitoring
}

\author{
Ramadhani Sinde $^{1 *}$, Shubi Kaijage ${ }^{2}$ and Karoli Njau ${ }^{3}$ \\ $\mathrm{PhD}$ student, Nelson Mandela African Institution of Science and Technology, Arusha, Tanzania ${ }^{1}$ \\ Senior Lecturer, Nelson Mandela African Institution of Science and Technology, Arusha, Tanzania ${ }^{2}$ \\ Professor, Nelson Mandela African Institution of Science and Technology, Arusha, Tanzania ${ }^{3}$
}

Received: 27-December-2019; Revised: 20-February-2020; Accepted: 24-February-2020

(C)2020 Ramadhani Sinde et al. This is an open access article distributed under the Creative Commons Attribution (CC BY) License, which permits unrestricted use, distribution, and reproduction in any medium, provided the original work is properly cited.

\begin{abstract}
Monitoring the forest's weather has been essential to living things over the years. Currently, there is a shortage of information on real-time temporal and spatial environmental conditions of the forest that drive forest health condition. This work focuses on the sensing of humidity and temperature as weather data from the forest. Unlike the traditional systems used to collect weather information, the use of wireless sensor network (WSN) gives real-time data capture from every point of the forest. However, the WSN faces, the number of challenges, including low bandwidth, low power, and short battery lifespan. In this situation, batteries cannot be replaced since nodes are deployed in an inaccessible area. In order to prolong the network lifetime and reduce the network delay, we propose Zone based Clustering (ZbC) scheme and efficient routing to find the best path between source and cluster head. Initially, we deploy sensor nodes in three coronas namely C1, C2 and C3. We place the sink node at the center of the coronas. Based on the center point of the corona, we split each corona into four partitions each with three zones. Our work composed of two phases such as ZbC and Routing. In the first phase, we reduce energy consumption in data aggregation via $\mathrm{ZbC}$ scheme. In $\mathrm{ZbC}$ scheme, the hybrid Particle Swarm Optimization (PSO) and Affinity Propagation (AP) algorithm are utilized. Network delay is reduced in the second phase using Ant Colony Optimization (ACO) and FireFly Algorithm (FFA). Simulation results confirm that our proposed solution achieves a higher network lifetime up to $30 \%$, reduces delay up to $35 \%$ and enhances throughput compared to the existing cooperative Time Division Multiple Access (cTDMA), Dynamic Random Allocation (DRA) and improved Artificial bee colony (iABC) methods.
\end{abstract}

\section{Keywords}

Wireless sensor network, Coronas, Zone based clustering, Environmental monitoring, Routing, Forests.

\section{Introduction}

The importance of conserving forests has been a massive motivation for this research. Forests play an essential role in preventing global warming by absorbing greenhouse gases and building sustainable societies. Forests have a variety of functions, such as land conservation, securing of water sources, control of climate change, and creation of natural environs essential to human existence and regulating the temperature of the atmospheric environment. There have been many changes in the field of wireless technology in the past decade, one of which is undoubtedly the introduction of WSN.

*Author for correspondence

36
Over the past few years, WSN has become widely accepted in several applications including security surveillance of the battlefield, environmental monitoring, wildlife habit monitoring, fire detection, etc. [1], as a useful productivity tool, with energy awareness and routing protocols becoming more and hotter research area for many researchers. WSN having self and auto-configuration of nodes and operating without any human intervention for years provided the opportunity to increase productivity and this kind of network ease to deploy and more flexible. This wide acceptance of wireless sensor network originated from its ability to provide the real-time data collection from remote areas and low production cost regardless of underlying networking technologies, allowed the development of the Internet of Things (IoT) and designing of several protocols to enhance routing of packets from source node to destination at a minimum level of energy 
consumption. Cluster formation is one of the essential approaches in WSN to reduce energy consumption. Clustering is a method of combining sensor node into clusters with a cluster head. A bioinspired algorithm such as hybrid PSO and Gravitational Search Algorithm (GSA) [2], Honey Bee (HB) algorithm [3], Genetic Algorithm (GA) [4] and Multi-Objective Evolutionary Algorithm (MOEA) [5] are used to select an optimum cluster head in WSN.

Routing is one of the significant processes to reduce delays during data transmission. Energy efficient scheduling based cross layer based adaptive routing protocol is used [6] for WSN. The proposed routing protocol comprises of two models that include the network model and radio energy model. Energy efficient clustering using multilevel routing is introduced [7] for WSN. Three different routing mechanisms are hierarchical routing using cluster identification, hierarchical routing using multi-hop and multi-level. Multi-Objective hybrid routing algorithm is proposed [8] for WSN. In this, two approaches are used that are secularization approach and lexicographical approach. Forwarding Area Division (FDS) and Adaptive Forwarding Area Selection Routing (AFASR) protocol [9] is proposed for WSN. Forwarding area division ensures all nodes within the same forwarding sub area. The main objective of this work is to develop a low cost and the real-time forest's environmental monitoring using energy efficient WSN. We use WSN since it proved to be an effective requirement for the continuous monitoring of the hostile regions such as sudden volcanic eruptions, forest fires, floods, etc. We implemented $\mathrm{ZbC}$ scheme and a proper routing protocol to reduce energy consumption and network delay in the system operation respectively.

\section{Related works}

Bayo et al. [10] have developed an early detection and monitoring of wildfire using low-rate wireless personal area network (LR-WPAN) system in the mesh topology. Their system designed to take measurements of different parameters from the different heights of the tree depending on the relief of the forest. For the achievement of early detection of the wildfire [10], proposed to collect some critical environmental data such as barometric pressure, temperature, light intensity, relative humidity, soil moisture, and smoke. In order to reduce power consumption, the author introduced the analogue switches to disconnect the sensor nodes when the system is in sleep mode. This approach does not consider the randomness deployment of nodes with unknown locations and the size of the forest. Lloret et al. [11] have designed an integrated fire detection system using a wireless sensor network and wireless local area network (WLAN). The integrated system detects the presence of fire by using sensor nodes with an IP-based camera. The sensor alarm will propagate to the server via the wireless network when the nodes discover a fire. The central server selects the closest wireless camera to the multisensor, and it sends a request for it to retrieve real-time figures from the region. Although this study integrates sensory data with the image the main advantage, still this system might not be compatible with the harsh environments like most the forests.

Aslan et al. [12] have developed a comprehensive framework for the use of wireless sensor networks for forest fire detection and monitoring. Their framework includes the wireless sensor network architecture, sensor deployment scheme, and clustering and communication protocols. The developed framework aims to detect a fire threat as early as possible. The researchers consider the factors that may affect the activity level of the network. They mentioned two factors as follows: the energy consumption of the sensor nodes and the environmental conditions.

Bouabdellah et al. [13] have proposed a solution using WSN to detect fire on the forest of Oran city in Algeria. They used two concurrent detection systems: the Canadian system and the Korean system. They conducted real experiments using a sensor Test-bed based on MICA-Z plate- form. In their study claim that most of the existing research choose to simulate their proposed solutions instead of doing experiments in real test-bed environments.

Hefeeda and Bagheri [14] have presented the design and evaluation of wireless sensor network for forest fire detection. They presented forest fire modeling by analysing the Fire Weather Index (FWI) System. Their analysis helped to show how the different components can be used to design efficient fire detection systems. Also, they implemented a distributed algorithm to solve the coverage problem in WSN. They also claimed that the proposed algorithm achieves various coverage degrees at different subareas of the forest. Finally, they presented a simple data aggregation scheme based on the FWI System. The main contribution of this approach is to provide unequal monitoring quality of forest zones. The presented design also maximizes 
the network lifetime by using data aggregation scheme in which it delivers only data that is of interest to the application.

Gao and Huang [15] have constructed a multi-sensorbased system with the $4 \mathrm{G}$ network to monitor fire in the forest. The system was designed based on Zigbee standard and 4G mobile communication. For the hardware, CC2530 was used to collect and transmit sensor data via the $4 \mathrm{G}$ network to the remote server. The use of multi-sensor with high data rate $4 \mathrm{G}$ network give early warning of the wildfire. The challenge of using the $4 \mathrm{G}$ network is that not all forest can have full coverage of high data rate mobile network.

Unfortunately, most of these studies consider the detection of a wildfire [15, 12, 16-20], only a few types of research focus on the primary sources on the fire in the forest. In this paper, we propose a collection of weather data from the forest such as temperature and humidity, since these two parameters will help to analyse and predict the occurrence of wildfire.

Díaz-Ramírez et al. [21] have proposed two WSN algorithms for forest fire detection. These are information fusion algorithms. The first one uses temperature, light, humidity sensors and a threshold method, whereas the Dempster-Shafer theory used in the second algorithm.

Some researches in [22-27], use solar power systems and a lithium-ion battery instead of using disposable batteries as power supplies. Zhu et al. [28] evaluated the impact of solar power systems in WSNs.

Thayananthan and Alzranhi [29] have proposed the enhancement of energy conservation technologies in WSN. In their method, they used a combination of routing protocols and solar power to enhance energy conservation. Their approach increases the lifetime of the sensor node and the battery life.

Frezzetti et al. [30] have presented the development of a solar energy harvesting mechanism for WirelessHART sensor node using photovoltaic (PV) cell array, controller, converter, and UltraCapacitor (UC).

Baranov et al. [31] have presented a hybrid power supply to reduce the power consumption of the node without degrading the sensing capabilities. In this study, they are integrated solar energy, wind and electrochemical $\mathrm{CO}$ sensor.

All the researches based on solar systems can improve the lithium-ion battery life and ensure the business continuity of the system. But for the implementation of the solar power system still needs to redo the experimentation on the operation of the charge and discharge control to make sure the command and estimation of battery power accuracy fulfil the system requirements. Most of the researchers did not pay much attention to the temperature and relative humidity as sources of wildfire, rather their focuses based on the smoke. It might be too late and very risky to detect smoke in the forest as this is a premature level of uncontrolled fire in the forest. Therefore, this work proposed to collect and analyze variations of environmental data. The collected environmental data can help to predict the wildfire by considering extensively rises in temperature.

This research intends to develop low cost and a realtime monitoring $\mathrm{WrSN}$ for collecting temperature and humidity data in Forest. Some of the researches did not consider the cost implications of their proposed systems and most of them focus on wildfire monitoring only not the energy constraints of the sensor nodes.

\section{Proposed work}

Our network comprises of static sensor nodes and a sink node as depicted in Figure 1.

\subsection{Clustering phase}

In WSN, clustering plays a vital role in terms of energy consumption, since it minimizes energy consumption during data aggregation. To overthrow this problem, we pursue $\mathrm{ZbC}$ scheme that forms an efficient cluster in each zone using hybrid PSO [32] and AP [33] algorithm. The shortcoming of AP algorithm is the number of exemplar selection is not effective. To overthrow this problem, we combined PSO with AP.

3.1.1PSO based exemplar selection

PSO algorithm operates based on the swarming nature of flocks of birds. In our work, we pursue PSO to choose optimum exemplar for AP. PSO computes fitness function for subsequent metrics including node degree, residual energy and distance. PSO selects exemplar in each zone to form effective clusters. PSO comprises of three phases that are initialization, fitness function evaluation, Global best $\left(G_{b}\right)$ and Local best $\left(L_{b}\right)$ computation. 


\section{a. Initialization}

In PSO, each sensor node is considered a particle that is associated with initialize position, node degree, residual energy and distance.

\section{b. Fitness function computation}

In this phase, PSO computes fitness function for each node using succeeding parameters such as node degree, residual energy and distance that are explained as follows:

\section{Node degree}

Node degree is described as the number of neighbor nodes connected to the particular node. This metric elects node having the highest node degree that can communicate with more number of neighbour node that tends to increase in network performance. Node degree is represented as ' $N_{D}$ ' that can be conveyed as follows:

$N_{D}=N_{c}\left(N_{i}\right)$ node $N_{i}$.

Where $N_{c}\left(N_{i}\right)$ represents neighbour count of

\section{Residual energy}

Residual energy is described as the difference between total primary energy and consumed energy. Herein, residual energy metric is used to elect the highest energy node to avoid frequent death of sensor node in the network. It is represented as ' $E_{r}$ ' that is expressed as follows:

$E_{r}=E_{p}-E_{c}$
Where $E_{p}$ represents total primary energy and $E_{c}$ represents consumed energy.

\section{Distance}

The distance parameter is referred to as the distance between sensor and sink node. This metric also involved in network delay, since the distance is directly proportional to the delay. It is represented as ' $D_{i, s}$ 'follows:

$D_{i, s}=\sqrt{\left(\left(N_{s, x}-N_{i, x}\right)^{2}+\left(N_{s, y}-N_{i, y}\right)^{2}\right)}$

Where, $N_{s, x}, N_{s, y}$ represents the position of sink node and $\left(N_{i, x}, N_{i, y}\right)$ represents the position of a sensor node.

By means of above metrics PSO computes fitness function for exemplar using below expression,

$f_{e}=\sigma_{1}\left(E_{r}\right)+\sigma_{2}\left(D_{i, s}\right)+\sigma_{3}\left(N_{D}\right)$

Where, $\sigma_{1}, \sigma_{2}$ and $\sigma_{3}$ are weightage parameters.

\section{Global best and local best computation}

After computing fitness function for each node, this phase compares each node local best ' $L_{b}$ ' with other nodes to find the global best ' $G_{b}{ }^{\prime}$ node. This process is functioned until stopping criterion reached. Finally, the optimum node with the highest fitness value is elected as an exemplar for the AP process.

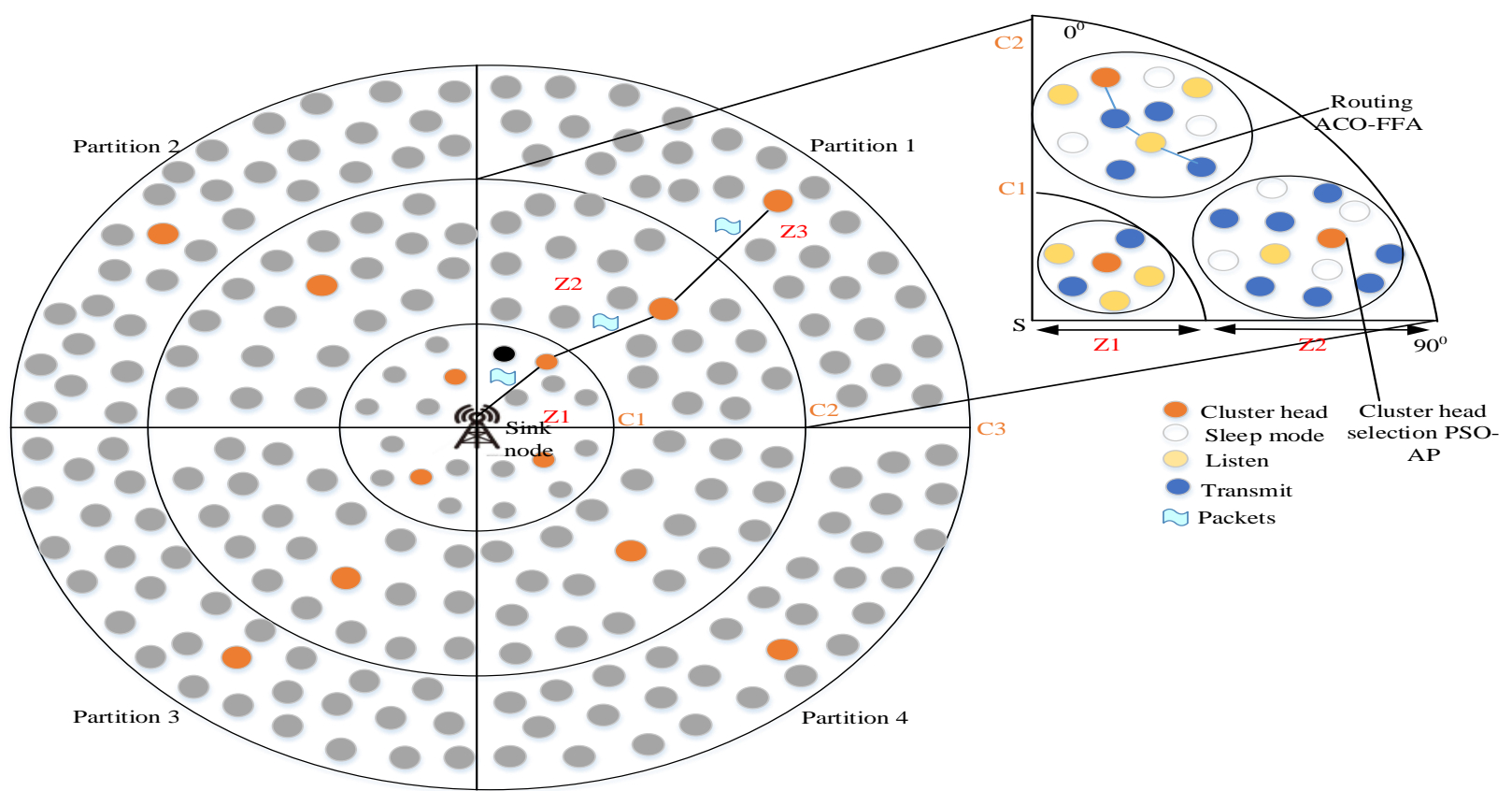

Figure 1 Architecture for proposed work 


\subsubsection{AP cluster formation}

Based on selected exemplar AP forms clusters, here exemplar nodes are elected by computing fitness function for each node. Using selected ' $k$ ' exemplar, AP forms clusters using similarity between sensor node $N_{i}$ and exemplar $N_{e}$ that is computed using the following expression,

$$
\operatorname{Sim}\left(N_{e}, N_{i}\right)=\left|N_{e}-N_{i}\right|^{2}
$$

Where $\left|N_{e}-N_{i}\right|^{2}$ represent the similarity between $N_{i}$ and $N_{e}$ in terms of Euclidean distance. AP has two matrices that are responsibility matrix and available matrix. Responsibility matrix and available matrix are updated using computed similarity. Responsibility matrix ' $R_{m}$ 'is updated as follows:

$$
\begin{gathered}
R_{m}\left(N_{e}, N_{i}\right)=\operatorname{Sim}\left(N_{e}, N_{i}\right)-\max _{i^{\prime} \neq i}\left(A\left(e, i^{\prime}\right)+\right. \\
\left.\quad s\left(e, i^{\prime}\right)\right)
\end{gathered}
$$

Where, $A\left(e, i^{\prime}\right)$ represents the availability matrix that is expressed as follows:

$$
\begin{aligned}
& A\left(e, i^{\prime}\right)=\min \left(0, R_{m}\left(N_{e}, N_{i}\right)\right) \\
& +\sum_{i^{\prime} \neq e, i} \max \left(0, R_{m}\left(N_{e}, N_{i}\right)\right)
\end{aligned}
$$

Using the above two equation responsibility and availability matrix is computed for each sensor node and exemplar node. This process is repeated until possible clusters are formed. Our proposed clustering hybrid algorithm forms effective clusters in each zone that reduces energy consumption through data aggregation.

\subsection{Routing phase}

Network delay is more in WSN due to inefficient path selection between source and the sink node. To overthrow the network delay problem, ACO and FFA algorithm-based routing are proposed. Multipath selection reduces delay in data transmission between source Cluster Member (CM) and exemplar. Hence, we first select multiple paths between $\mathrm{CM}$ and exemplar using ACO. From selected multipath, the optimum path is selected using FFA.

\subsubsection{ACO algorithm}

ACO algorithm [34] works based real ants behaviour. ACO algorithm computes fitness function using subsequent parameters such as hop count, bandwidth, residual energy and distance. In ACO multipath is selected based on the pheromone value of each node. Pheromone value is updated in each iteration to discover the best path between source $\mathrm{CM}$ and exemplar. Pheromone value is computed using the following parameters that are explained as follows:

\section{a. Hop count}

Hop count is described as the number of nodes between source CM and exemplar. This metric is used to reduce delay in data transmission since delay tends to increase the energy consumption of the sensor node. It is represented as ' $H_{c}$ ' that is explained as follows:

$H_{c}=N_{c}\left(N_{C M_{i}}, N_{e}\right)$

Where, $N_{c}\left(N_{C M_{i}}, N_{s}\right)$ represent hop count between source cluster member node $N_{C M_{i}}$ and exemplar $N_{e}$.

\section{b. Distance}

The distance parameter is referred to as the distance between source cluster member and exemplar node. This metric also involved in network delay, since the distance is directly proportional to the delay. It is represented as ' $D_{C M_{i, e}}$ ' that can be computed as same as equation 3 .

\section{c. Bandwidth}

Bandwidth parameter is considered to know the capacity of the link. Typically, bandwidth is measured in terms of bits per second. Bandwidth metric is used to elect the best path to route the sensed data. It is represented as ' $B_{p}$ ' that is expressed as follows:

$B_{p}=\frac{b_{n}}{s}$

Where $b_{n}$ indicates the number of bits and $s$ indicates seconds.

In proposed ACO algorithm ants are working based on two rules. At first, ants selects paths in initial rule and then it finds the optimum path in revised rule.

\section{Initial rule}

In this rule probability of selecting a path is computed using pheromone. Pheromone function is computed using fitness value and pheromone intensity. At first, ACO compute fitness values for each node to elect multiple paths between source CM and exemplar node. Fitness value ' $V_{f}$ ' is computed using equation 10 ,

$V_{f}=\sum E_{r}+H_{c}+B_{p}+\frac{1}{D_{i, s}}$

Above expression composed of all computed parameters such as residual energy, hop count, distance and bandwidth. Utilizing the computed fitness value, ACO further computes pheromone for each path for data transmission. The pheromone function ' $P_{f}$ ' is computed using the succeeding expression:

$P_{f}=\frac{\tau_{i, j}{ }_{* V_{f}}^{\beta}}{\sum_{i=0}^{n} \tau_{i, j} \alpha_{* V_{f}}{ }^{\beta}}$

Where, $\tau_{i, j}$ indicates pheromone intensity, $\alpha$ and $\beta$ are control parameters. Probability of selecting a path between source CM and exemplar node is expressed as follows:

$\rho_{k}=\frac{\alpha+\left[P_{f}\right] * \beta}{\sum_{i=0}^{n} \alpha+\left[P_{f}\right] * \beta}$ 
Equation 12 indicates the probability of selecting path as a transmission path between source $\mathrm{CM}$ and exemplar node.

\section{Revising rule}

In this rule, the pheromone is updated in each iteration using the following expression,

$\tau_{i, j}(t+1)=(1-\varepsilon) \tau_{i, j}(t)+\varepsilon \Delta \tau_{i, j}(t)$

Where $\varepsilon$ represents local pheromone corrosion and $\Delta \tau_{i, j}(t)$ represents pheromone enhancement. Using updating pheromone values in each iteration, ACO discovers multiple paths between source $\mathrm{CM}$ and exemplar node.

\subsubsection{Firefly}

From the selected multiple paths, FFA [35] elects the best path between source CM and exemplar. FFA works on the principle of flashing lights of fireflies. Here, FFA computes fitness function for succeeding metrics such as packet delivery ratio, expected delay and load.

\section{a) Packet delivery ratio}

Packet delivery ratio is described as the ratio between the number of packets successfully transmitted by the source node and number packets successfully received by the exemplar node. It is expressed ' $\mathbb{d}_{r}$ ' as follows:

$\mathbb{d}_{r}=\frac{p_{s}}{p_{r}}$

Where $p_{s}$ represents packets successfully transmitted and $p_{r}$ indicates packets successfully received.

b) Expected delay

Expected delay is described as the time required to transmit data from the source to the destination node. This parameter is taken to measure the delay in the selected path since it affects the energy consumption of the network. It is represented as ' $\mathbb{E}_{d}$ ' that is expressed as follows:

$\mathbb{E}_{d}=T\left(N_{C M_{i}}, N_{e}\right)$

Where, $T\left(N_{C M_{i}}, N_{e}\right)$ represents the time required to transmit data between source $\mathrm{CM}$ node $N_{C M_{i}}$ and exemplar node $N_{e}$.

\section{c) Load}

Load parameter is used to measure the load of each sensor node in the network. Load parameter is directly proportional to the energy consumption; hence the load of the sensor node increases automatically energy consumption also increase. This parameter is expressed as ' $N_{L}$ ' that can be conveyed as follows:

$N_{L}=L\left(N_{i}\right)$

Where $L\left(N_{i}\right)$ represents load of the node $N_{i}$.

FFA computes fitness function for each node to find the effective path between source CM and exemplar node. Fitness function $f(x)$ is expressed as follows:

$f(x)=\sum \frac{1}{E_{d}+N_{L}}+D_{r}$

The light intensity of each node is computed using the above explained fitness function $f(x)$. Light intensity ' $I_{l}$ ' is expressed as follows:

$I_{l}=I_{o} e^{-\gamma r}$

Where $I_{o}$ indicates original light intensity.

The attractiveness of FFA is computed using below expression,

$\omega=\omega_{0} e^{-\gamma r^{2}}$

Where $\omega_{0}$ indicates attractiveness at $\mathrm{r}=0$. Here, firefly moves to the best position from $\mathrm{i}$ to $\mathrm{j}$ by using below expression,

$$
\begin{aligned}
& N_{i}=N_{i}+\omega_{0} e^{-\gamma r^{2}}\left(N_{j}-N_{i}\right)+ \\
& \delta\left(\text { rand }-\frac{1}{2}\right)
\end{aligned}
$$

By using above function firefly discover the optimum path between source $\mathrm{CM}$ and exemplar node. From the above process, our work effectually reduces network delay that in turn enhances network lifetime.

\section{Simulation parameters}

Our proposed work is implemented in Network Simulator 3 (NS3) tool that is implemented in the Ubuntu operating system. NS3 is a discrete event simulator that provides simulations of different types of network; hence we preferred this simulator for proposed energy efficient sleep scheduling in WSN environment. Figure 2 shows the selection of optimum path using ACO and FFA. Table 1 shows the simulation parameter.

Table 1 Simulation parameter

\begin{tabular}{ll}
\hline Parameters & Value \\
\hline Simulation area & $1000 * 1000 \mathrm{~m}$ \\
Number of sensor nodes & 100 \\
Number of the sink node & 1 \\
Initial energy of the node & $700 \mathrm{~J}$ \\
Number of packets & 200 \\
Number of retransmission & Max 7 \\
Packet size & $1024 \mathrm{MB}$ \\
\hline
\end{tabular}




\begin{tabular}{ll}
\hline Parameters & Value \\
\hline Packet interval & $0.1 \mathrm{~s}$ \\
Sensor communication range & $100 \mathrm{~m}$ \\
Data rate & $20 \mathrm{Mbps}$ \\
Number of slots & 16 \\
Slot duration & $10 \mu \mathrm{s}$ \\
Simulation time & $10 \mathrm{~s}$ \\
\hline
\end{tabular}

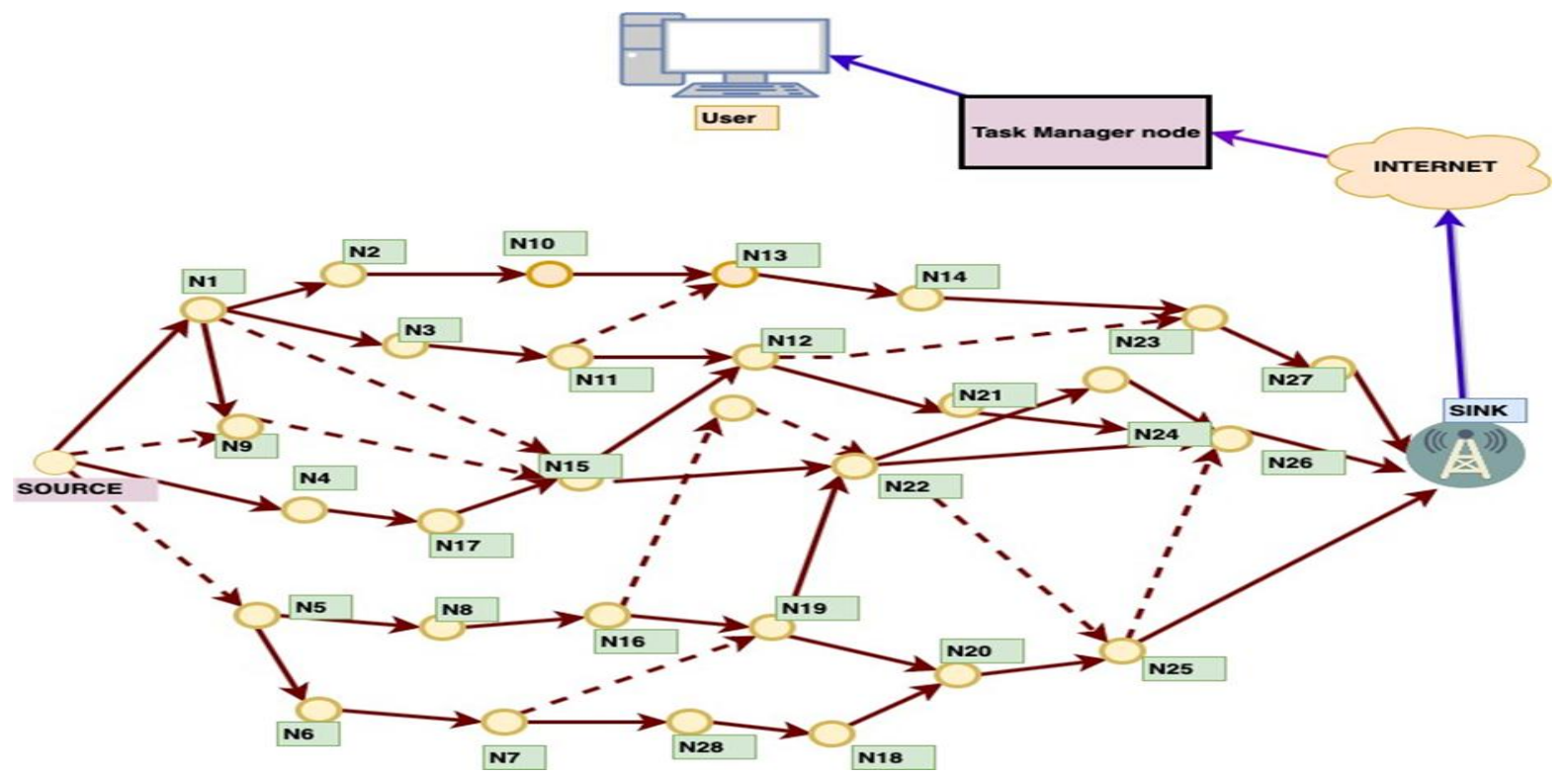

Figure 2 Selection of optimum path using ACO and FFA

\section{Results and discussion}

In this section, we describe the evaluation of proposed work through following metrics such as network lifetime, energy consumption, throughput and delay. This section is further divided into two sections that are performance metrics and comparative analysis. We compare our proposed work performances with existing methods.

\subsection{Performance metrics}

We evaluate the performance of our work using performance metrics such as network lifetime, energy consumption, throughput and delay that are summarized as follows:

5.1.1Network lifetime

Network lifetime metric is used to measure the lifetime of the sensing network. Typically, network lifetime is referred to as the time at which the first node dies in the network. It is also defined as an operational time of node during which it can able to perform the allocated task. Network lifetime is represented as ' $n_{\ell}$ ' that can be conveyed as follows: $n_{\ell}=\frac{\jmath_{\mathcal{E}}-w_{e}}{c_{p}+a_{r} \mathcal{R}_{e}}$
Where $\mathcal{J}_{\mathcal{E}}$ denote initial energy of the network, $w_{e}$ denotes wasted energy, $C_{p}$ denotes continuous power consumption of the network, $\mathfrak{a}_{r}$ represents average sensor reporting rate and $\mathcal{R}_{e}$ represents estimated reporting energy.

5.1.2Throughput

Throughput metric is described as the number of packets successfully received by the receiver in the given amount of time. It is represented as ' $\mathcal{T}$ ' that is conveyed as follows:

$\mathcal{T}=\frac{\sum_{i=1}^{n} \mathfrak{p}_{i} * \mathfrak{p}_{\ell}}{s(t)}$

Where, $\mathfrak{p}_{i}$ represents the number of packets in node ' $i$ ', $\mathfrak{p}_{\ell}$ denotes data packet length and $s(t)$ represents simulation time.

\subsubsection{Delay}

Delay is defined as the time required to deliver sensed data from the source to the destination node. It is represented as ' $\mathfrak{D}$ ' that is conveyed as follows:

$\mathfrak{D}=\frac{T_{s}}{T_{d}}$

Where $T_{s}$ represents the time required to send data and $T_{d}$ represents data received by the destination node. 


\subsection{Comparative analysis}

In this section, we demonstrate a comparison of our proposed work performances with the existing methods iABC [36], cTDMA [37] and DRA [38] methods.

\subsubsection{Analysis on network lifetime}

Network lifetime metric is a significant metric to analysis performance of proposed work. It demonstrates the efficiency of our proposed work in terms of the lifetime of the network. In our work, the network lifetime metric is measured with the number of nodes or simulation time.

Figure 3 shows our proposed work achieves high network lifetime compared to the existing methods of iABC and cTDMA. Our method achieves a maximum of 6760rounds network lifetime in the presence of 100 nodes. Meanwhile, the existing method $\mathrm{iABC}$ and cTDMA achieves minimum network lifetime for 100 nodes. Here, cTDMA method has a minimum lifetime of 4500 rounds for 100 nodes.

Figure 4 illustrates network lifetime with respect to the simulation time that demonstrates Network lifetime decreases dramatically when simulation time increases. Our method achieves maximum average network lifetime of 6710 rounds for $10 \mathrm{sec}$ of simulation time whereas existing method $\mathrm{iABC}$ and cTDMA methods minimum average network lifetime 5620 rounds and 4860 rounds for $10 \mathrm{sec}$ of simulation time respectively.

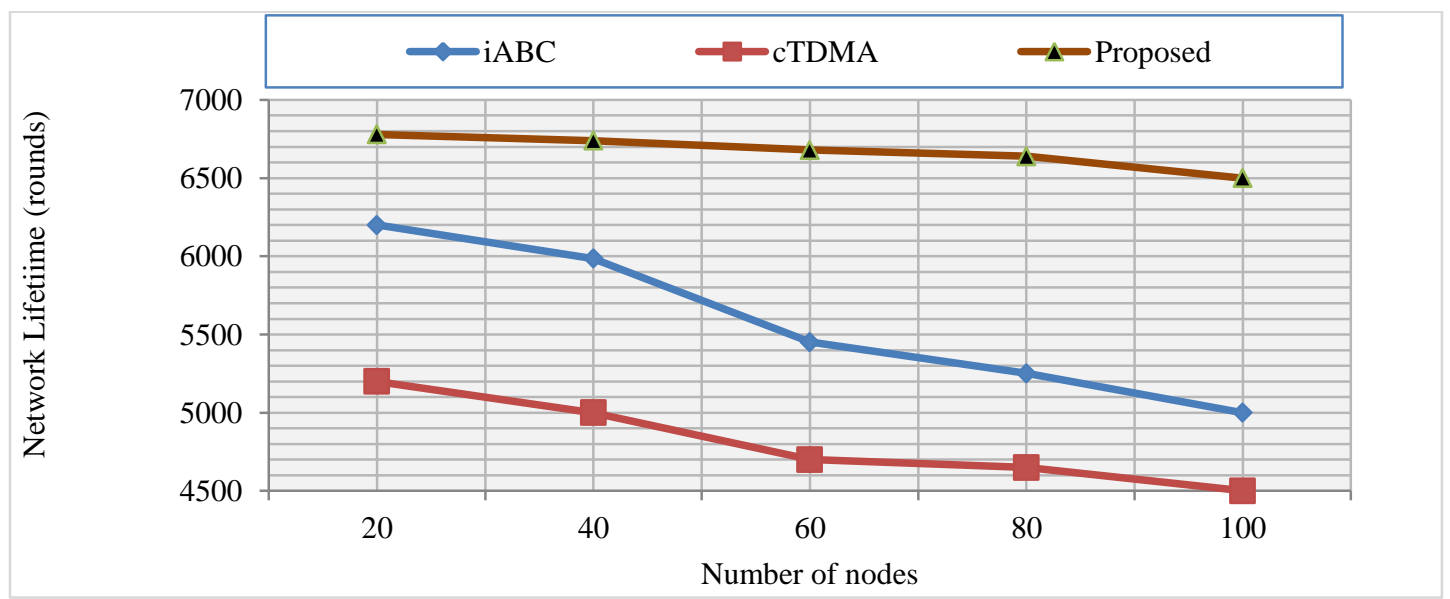

Figure 3 Comparison of network lifetime vs. number of nodes

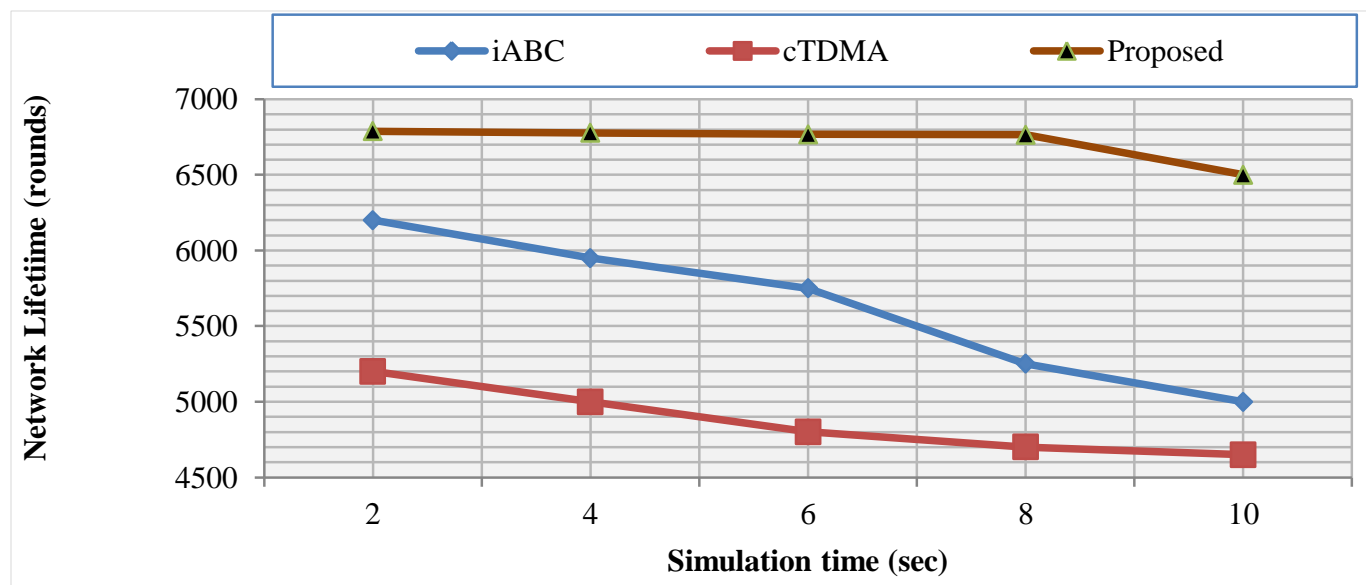

Figure 4 Comparison on network lifetime vs. simulation time 


\subsubsection{Analysis of throughput}

Throughput metric related to the performance of the proposed network. Hence, throughput increases as much as possible in our work. In performance evaluation throughput metric is measured using the number of nodes or number of rounds. Figure 5 describes our proposed work produces high throughput compared to the existing methods such as $\mathrm{iABC}$ and cTDMA. In existing methods like $\mathrm{iABC}$ and cTDMA losses data packets in transmission due to poor path selection between source and the sink node. Our work achieves a maximum of $90 \%$ throughput for 100 nodes. iABC achieves a maximum of $69 \%$ and cTDMA method achieves a maximum of $78 \%$ for 100 nodes

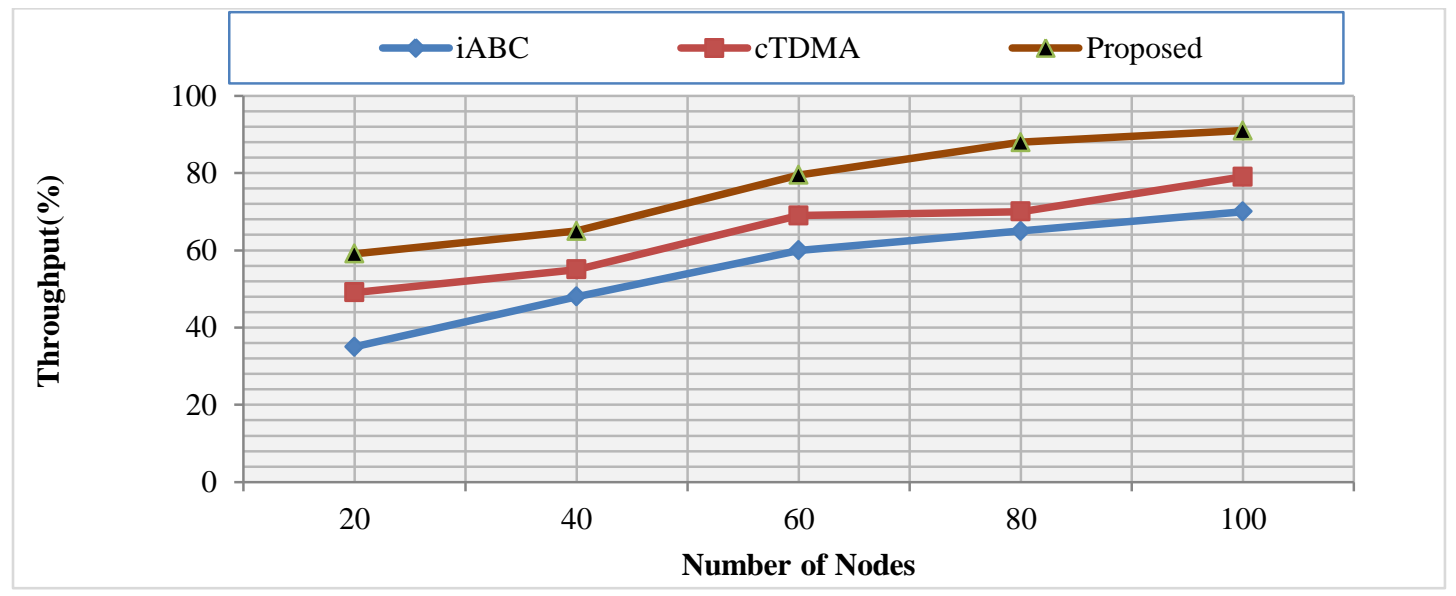

Figure 5 Comparison on throughput

\subsubsection{Analysis of delay}

Delay metric is significant for improving the lifetime of the network since it is directly proportional to the energy consumption and network lifetime metric. Thus, our work reduces delay as much as possible. Usually, the delay metric is measured using the number of nodes or number of rounds.

Figure 6 demonstrates that the delay of proposed work is minimal compared to the existing methods $\mathrm{iABC}$ and DRA. If the number of node increases, automatically delay also increases. Our method achieves a minimum of $235 \mathrm{msec}$ for 100 nodes. DRA method has poor data transmission since it has a maximum delay of $445 \mathrm{msec}$ for 100 nodes.

The monitoring of forest environmental condition using cluster based WSN, the results from simulation analysis have been compared with the existing methods iABC, cTDMA, and DRA. The experiment carried out to evaluate our solution on the following metrics: network lifetime, energy consumption, throughput and delay. Our solution consumes the minimal energy and hence has the highest network lifetime compared with cTDMA and iABC. In this simulation, cTDMA method is the highest energy consumer with a minimum lifetime of 4500 rounds for 100 nodes. The proposed solution achieves the highest throughput compared to the $\mathrm{iABC}$ and cTDMA methods. The existing solutions have fewer throughputs due to poor paths selection between the source and the sink node. In our proposed solution, we first select multiple paths between CM and exemplar using ACO. From selected multipath, the optimum path is selected using FFA. Due to the optimal path selection, our work achieves a maximum of $90 \%$ throughput for 100 nodes. Whereas $\mathrm{iABC}$ and cTDMA methods achieve a maximum throughput of $69 \%$ and $78 \%$ respectively.

The above comparison clearly shows our work transmits packets with minimum delay. Since we propose efficient routing and $\mathrm{ZbC}$ scheme. The delay is reduced through effective path selection between source and destination. Here, ACO selects multiple paths between source and destination and FFA selects the best path among multiple paths that in turn reduces delay effectively. Our method achieves a minimum of $235 \mathrm{msec}$ for 100 nodes. DRA method has poor data transmission since it has a maximum delay of $445 \mathrm{msec}$ for 100 nodes. From the above comparison, we conclude that our method achieves better data transmission with minimum delay in the network. 


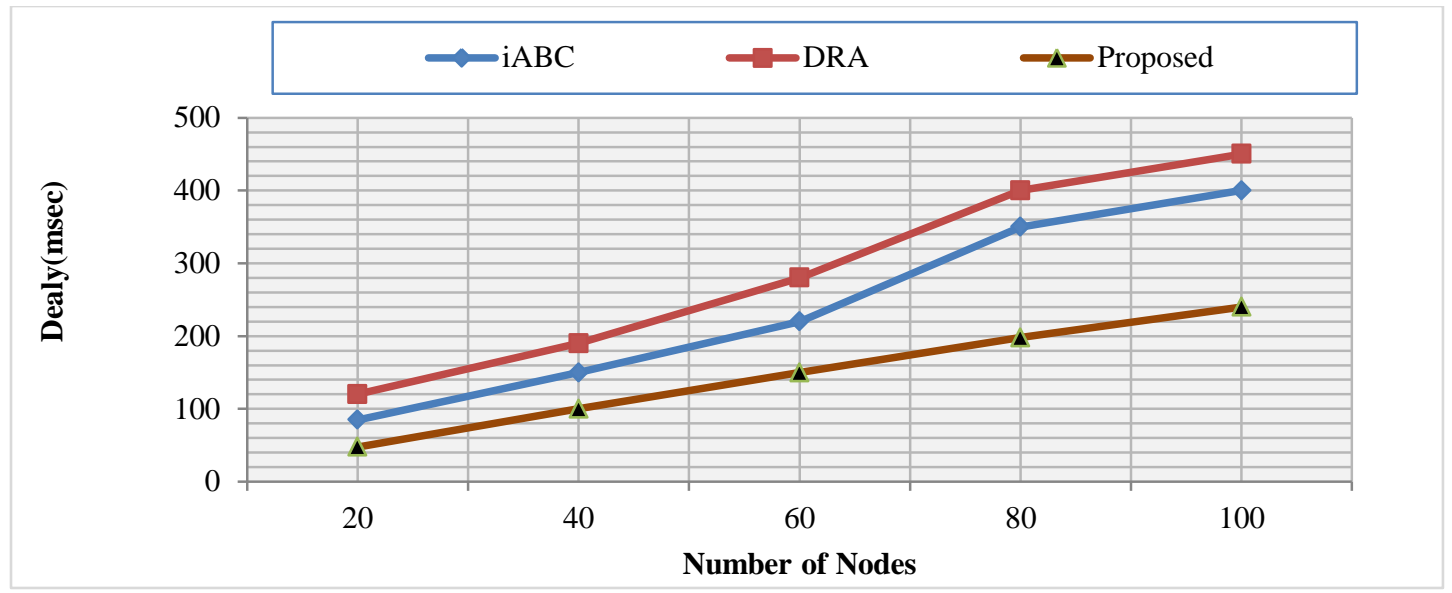

Figure 6 Comparison of delay

\section{Conclusion and future work}

In this research, a cluster-based network to collect forest's climatological data using WSN has proposed. To enhance the network lifetime and reducing the transmission delay in this network two novel methods have proposed. The first method aims to reduce energy consumption during data aggregation through $\mathrm{ZbC}$ scheme that comprises hybrid $\mathrm{PSO}$ and $\mathrm{AP}$ algorithm. In this case, PSO computes fitness function for following metrics energy factor and node degree. The energy factor is a combination of residual energy and distance between the source and sink node. The second method is to reduce the data transmission delay. In this case, the ACO algorithm is used to choose multiple paths between the source and cluster head. Here, the ACO algorithm considers following metrics to compute fitness function such as residual energy, the distance between the source and the destination node, hop count and bandwidth. From the selected multiple paths, the best one between the source and cluster head is selected using the firefly algorithm. Firefly algorithm considers succeeding metrics such as expected delay, packet delivery ratio and load. The climatological data will be stored in a database for further analysis. In this work, the performance of the proposed solution was evaluated by implementing it in a simulation environment. Simulations are conducted using ns 3.26 and to get precise plots, the confidence interval is taken. The simulation results show that the proposed solution performs better considering metrics of energy consumption, network lifetime, transmission delay, and network throughput. From these comparisons, it is clear that the proposed work is more efficient than existing methods such as iABC, cTDMA and DRA. Our experiment was limited to homogeneous and stationary deployed sensor nodes. In this work, we used only 100 nodes for simulation.

\subsection{Recommendation}

This research could be done differently by extending it to monitor and detect wildfire with enough money and time. Different types of sensor nodes for soil moisture, pressure, smoke would be needed to predict fire. The proposed solution can be integrated with a framework used for fire detection. The detection of wildfire might be done by setting threshold values of the weather parameters etc. Implementation of the data science techniques and machine learning concept would be better for the analysis of the collected weather data and fire prediction. Also, this research can be integrated with other systems for precision agriculture in huge farms, since network lifetime and delay have been carefully considered. The number of nodes deployed in the present study was relatively small. A more significant amount of sensor nodes would probably give a clearer picture of the situation. Therefore, future studies may attempt to use a larger number of sensor nodes. Also, the deployment issues, network security and data quality still open for further research. As it is known that, the forest is a large and harsh area which is sometimes is not accessible for nodes deployment. The random deployment of sensors by dropping them from the helicopter or drones would fit the purpose than trying to use a deterministic approach.

\section{Acknowledgment}

The authors acknowledge DEMIS project funded by DAAD-German and African Development Bank (AFDB) for funding this research and anonymous reviewers for their contribution. 
Conflicts of interest

The authors have no conflicts of interest to declare.

\section{References}

[1] Thakur A, Prasad D, Verma A. Deployment scheme in wireless sensor network: a review. International Journal of Computer Applications. 2017; 163(5):12-5.

[2] Morsy NA, AbdelHay EH, Kishk SS. Proposed energy efficient algorithm for clustering and routing in WSN. Wireless Personal Communications. 2018; 103(3):2575-98

[3] Ahmad M, Ikram AA, Wahid I, Inam M, Ayub N, Ali $\mathrm{S}$. A bio-inspired clustering scheme in wireless sensor networks: BeeWSN. Procedia Computer Science. 2018; 130:206-13.

[4] Yuan X, Elhoseny M, El-Minir HK, Riad AM. A genetic algorithm-based, dynamic clustering method towards improved WSN longevity. Journal of Network and Systems Management. 2017; 25(1):2146.

[5] Elhabyan R, Shi W, St-Hilaire M. A pareto optimization-based approach to clustering and routing in wireless sensor networks. Journal of Network and Computer Applications. 2018; 114:57-69.

[6] Singh R, Verma AK. Energy efficient cross layer based adaptive threshold routing protocol for WSN. AEU-International Journal of Electronics and Communications. 2017; 72:166-73.

[7] Muthukumaran K, Chitra K, Selvakumar C. An energy efficient clustering scheme using multilevel routing for wireless sensor network. Computers \& Electrical Engineering. 2018; 69:642-52.

[8] Kulkarni N, Prasad NR, Prasad R. Q-MOHRA: QoS assured multi-objective hybrid routing algorithm for heterogeneous WSN. Wireless Personal Communications. 2018; 100(2):255-66.

[9] Hong C, Zhang Y, Xiong Z, Xu A, Chen H, Ding W. FADS: Circular/spherical sector based forwarding area division and adaptive forwarding area selection routing protocol in WSNs. Ad Hoc Networks. 2018; 70:121-34.

[10] Bayo A, Antolín D, Medrano N, Calvo B, Celma S. Early detection and monitoring of forest fire with a wireless sensor network system. Procedia Engineering. 2010; 5:248-51.

[11] Lloret J, Garcia M, Bri D, Sendra S. A wireless sensor network deployment for rural and forest fire detection and verification. Sensors. 2009; 9(11):8722-47.

[12] Aslan YE, Korpeoglu I, Ulusoy Ö. A framework for use of wireless sensor networks in forest fire detection and monitoring. Computers, Environment and Urban Systems. 2012; 36(6):614-25.

[13] Bouabdellah K, Noureddine H, Larbi S. Using wireless sensor networks for reliable forest fires detection. Procedia Computer Science. 2013; 19:794801.

[14] Hefeeda M, Bagheri M. Forest fire modeling and early detection using wireless sensor networks. Ad Hoc \& Sensor Wireless Networks. 2009; 7(3-4):169-224.
[15] Gao Z, Huang L. A forest fire monitoring and early warning system based on the technology of multisensor and multilevel data fusion. In 2nd international conference on electrical, computer engineering and electronics 2015(pp. 627-32). Atlantis Press.

[16] Al-Habashneh AA, Ahmed MH, Husain T. Reliability analysis of wireless sensor networks for forest fire detection. In international wireless communications and mobile computing conference 2011 (pp. 1630-5). IEEE.

[17] Hefeeda M, Bagheri M. Wireless sensor networks for early detection of forest fires. In international conference on mobile adhoc and sensor systems 2007 (pp. 1-6). IEEE.

[18] Liu Y, Gu Y, Chen G, Ji Y, Li J. A novel accurate forest fire detection system using wireless sensor networks. In seventh international conference on mobile Ad-hoc and sensor networks 2011 (pp. 52-9). IEEE.

[19] Son B, Her YS, Kim JG. A design and implementation of forest-fires surveillance system based on wireless sensor networks for South Korea mountains. International Journal of Computer Science and Network Security (IJCSNS). 2006; 6(9):124-30.

[20] Soliman H, Sudan K, Mishra A. A smart forest-fire early detection sensory system: another approach of utilizing wireless sensor and neural networks. In SENSORS, 2010 (pp. 1900-4). IEEE.

[21] Díaz-Ramírez A, Tafoya LA, Atempa JA, MejíaAlvarez P. Wireless sensor networks and fusion information methods for forest fire detection. Procedia Technology. 2012; 3:69-79.

[22] Dehwah AH, Elmetennani S, Claudel C. UD-WCMA: an energy estimation and forecast scheme for solar powered wireless sensor networks. Journal of Network and Computer Applications. 2017; 90:17-25.

[23] Dondi D, Bertacchini A, Brunelli D, Larcher L, Benini L. Modeling and optimization of a solar energy harvester system for self-powered wireless sensor networks. IEEE Transactions on Industrial Electronics. 2008; 55(7):2759-66.

[24] Horng GJ, Chang TY, Cheng ST. An effective nodeselection scheme for the energy efficiency of solarpowered WSNs in a stream environment. Expert Systems with Applications. 2014; 41(7):3143-56.

[25] Ibrahim R, Chung TD, Hassan SM, Bingi K, Salahuddin S. Solar energy harvester for industrial wireless sensor nodes. Procedia Computer Science. 2017; 105:111-8.

[26] Noh DK, Kang K. Balanced energy allocation scheme for a solar-powered sensor system and its effects on network-wide performance. Journal of Computer and System Sciences. 2011; 77(5):917-32.

[27] Yue R, Ying T. A novel water quality monitoring system based on solar power supply \& wireless sensor network. Procedia Environmental Sciences. 2012; 12:265-72.

[28] Zhu Y, Song J, Dong F. Applications of wireless sensor network in the agriculture environment monitoring. Procedia Engineering. 2011; 16:608-14. 
[29] Thayananthan V, Alzranhi A. Enhancement of energy conservation technologies in wireless sensor network. Procedia Computer Science. 2014; 34:79-86.

[30] Frezzetti A, Manfredi S, Pagano M. A design approach of the solar harvesting control system for wireless sensor node. Control Engineering Practice. 2015; 44:45-54.

[31] Baranov A, Spirjakin D, Akbari S, Somov A, Passerone R. POCO:'Perpetual'operation of CO wireless sensor node with hybrid power supply. Sensors and Actuators A: Physical. 2016; 238:112-21.

[32] Kaur T, Kumar D. Particle swarm optimization-based unequal and fault tolerant clustering protocol for wireless sensor networks. IEEE Sensors Journal. 2018; 18(11):4614-22.

[33] Fan Z, Jiang J, Weng S, He Z, Liu Z. Adaptive density distribution inspired affinity propagation clustering. Neural Computing and Applications. 2019; 31(1):43545.

[34] Liu X, He D. Ant colony optimization with greedy migration mechanism for node deployment in wireless sensor networks. Journal of Network and Computer Applications. 2014; 39:310-8.

[35] Mosavvar I, Ghaffari A. Data aggregation in wireless sensor networks using firefly algorithm. Wireless Personal Communications. 2019; 104(1):307-24.

[36] Mann PS, Singh S. Optimal node clustering and scheduling in wireless sensor networks. Wireless Personal Communications. 2018; 100(3):683-708.

[37] Lahane SR, Jariwala KN. Network structured based routing techniques in wireless sensor network. In international conference for convergence in technology (I2CT) 2018 (pp. 1-6). IEEE.

[38] Bhardwaj R, Kumar D. MOFPL: Multi-objective fractional particle lion algorithm for the energy aware routing in the WSN. Pervasive and Mobile Computing. 2019; 58.

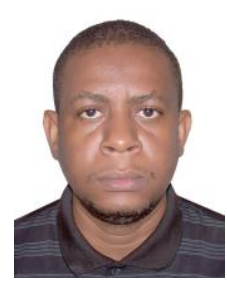

Ramadhani Sinde received the Bachelor degree in 2009, and Master's degree in 2011, both in Engineering and Technologies in Telecommunication specialized in Radio Engineering and Communication Systems and Equipment, from Moscow Technical University of Communication and Informatics. In 2013 he spent four months at Dhirubhai Ambani Institute of Information and Communication Technology as a visiting researcher under CV Raman scholarship. In 2016 he spent six months at Centre for Development of Advanced Computing in India for Postgraduate studies in mobile computing. He works as an assistant lecturer in the School of Computational and Communication Sciences and Engineering at Nelson Mandela African Institution of Science and Technology (NM-AIST). Currently, he under Doctorial Environmental Management Information System project undertaking $\mathrm{PhD}$ studies in the field of Information and Communication of Science and Engineering. His research interests include Computer Network, Mobile Computing, Modelling and
Performance Evaluation of Wireless Sensor Networks, wireless and mobile communication. Mr. Sinde has coauthored more than 10 papers in internationally refereed journals and conferences. $\mathrm{He}$ is a Queen Elizabeth Scholarship - Advanced Scholars Program alumni.

Email: sinder@nm-aist.ac.tz

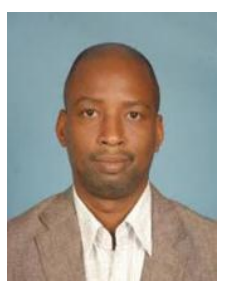

Shubi F. Kaijage obtained his Doctor of Engineerinng degree (Electronics and Information Eng.) from University of the Ryukyus, Okinawa, Japan, in March 2011. Since February 2014, he has been working with Nelson Mandela African Institution of Science and Technology (NM-AIST), Tanzania,, in the school of Computational and Communication Science and Engineering (CoCSE). From September 2011 to December 2013, Shubi worked as Post-Doctoral Research Fellow at the Research Center of Terahertz Technology, P.R. China. Shubi has vast experience in research of over 8 years in the areas of fiber optics, photonics and Terahertz technology, wireless sensor network and RFID technologies. Shubi has published over 25 scientific papers in International peer-reviewed journals and over 50 research works presented in various international conferences and workshops. Furthermore, he is recipient of numerous International award grants as recognition to his scholarly works. $\mathrm{He}$ is the member of International professional societies including the Institute of Electrical and Electronics Engineering (IEEE) and the Optical Society of America (OSA).

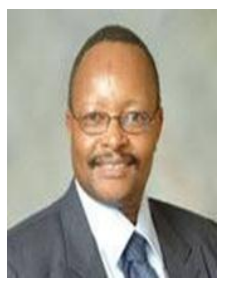

Karoli Nicholas Njau was born and raised on the slopes of Kilimanjaro, in Tanzania. Prof. Njau obtained his BSc. Hons. from the University of Dar es Salaam, Tanzania, majoring Process Engineering. He later did his Master degree in Chemical Engineering at the Norwegian Institute of Technology (NTH) Trondheim, Norway. Prof. Njau did his PhD studies at the Technical University Eindhoven, in the Netherlands. $\mathrm{He}$ is currently a Full Professor of the Department of Water and Environmental Science and Engineering at Nelson Mandela African Institution of Science and Technology (NM-AIST). His expertise in Environmental Toxicology, Environmental Mutagenicity, Environmental Hydrology, Spring Hydrology. On these topics he has published more than 100 peer-reviewed papers and conferences. Prof. Njau is an educator, researcher, and an international consultant on environmental issues. He has led many national and international research projects in the field for Water Resources and Environment. 